Yu-Fang Wang*, Jing-Jing Wei and Xiao-Hui Zhai

\title{
Crystal structure of catena-poly[ $\left(\mu_{2}-5\right.$-(tert-butyl)isophthalato- $\kappa^{4} O, 0^{\prime}: 0^{\prime \prime}$, $\left.O^{\prime \prime \prime}\right)\left(-4^{\prime}\right.$-(pyridin-4-yl)-2,2':6',2"'-terpyridine- $\left.{ }^{3} N, N^{\prime}, N^{\prime \prime}\right)$ manganese(II)], $\mathrm{C}_{32} \mathrm{H}_{28} \mathrm{~N}_{4} \mathrm{O}_{5} \mathrm{Mn}$
}

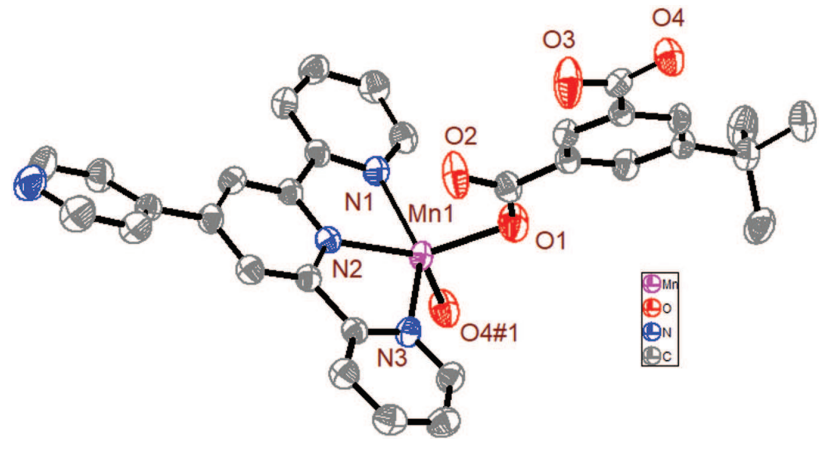

https://doi.org/10.1515/ncrs-2018-0189

Received May 29, 2018; accepted July 9, 2018; available online July 23, 2018

\section{Abstract \\ $\mathrm{C}_{32} \mathrm{H}_{28} \mathrm{~N}_{4} \mathrm{O}_{5} \mathrm{Mn}$, monoclinic, $P 2_{1} / c$ (no. 14), $a=8.777$ (3) $\AA$, $b=30.975(11) \AA, \quad c=10.229(4) \AA, \quad \beta=105.412(7)^{\circ}$, $V=2680.9(16) \AA^{3}, Z=4, R_{\mathrm{gt}}(F)=0.0448, w R_{\mathrm{ref}}\left(F^{2}\right)=0.1136$, $T=296(2) \mathrm{K}$. \\ CCDC no.: 1854556}

The crystal structure is shown in the figure. Hydrogen atoms are omitted for clarity (symmetry code \#1: $x, y,-1+z$ ). Tables 1 and 2 contain details on crystal structure and measurement conditions and a list of the atoms including atomic coordinates and displacement parameters.

\section{Source of material}

A mixture of 5-(tert-butyl)isophthalic acid ( $\mathrm{H}_{2}$ tbip, $23 \mathrm{mg}$,

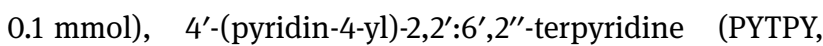
$32 \mathrm{mg}, 0.1 \mathrm{mmol}), \mathrm{Mn}(\mathrm{OAc})_{2} \cdot 4 \mathrm{H}_{2} \mathrm{O}(25 \mathrm{mg}, 0.1 \mathrm{mmol})$ and $\mathrm{H}_{2} \mathrm{O}(12 \mathrm{~mL}$ ) was stirred for $30 \mathrm{~min}$, and the $\mathrm{pH}$ value of the solution was adjusted to about 6 with $1 \mathrm{M} \mathrm{KOH}$. Then the mixture was transferred to a $25 \mathrm{~mL}$ Teflon-lined stainless

\footnotetext{
*Corresponding author: Yu-Fang Wang, College of Chemistry and Chemical Engineering, and Henan Key Laboratory of FunctionOriented Porous Materials, Luoyang Normal University, Luoyang, Henan 471934, P.R. China, e-mail: wangyf78@163.com

Jing-Jing Wei and Xiao-Hui Zhai: College of Chemistry and Chemical Engineering, and Henan Key Laboratory of Function-Oriented Porous Materials, Luoyang Normal University, Luoyang, Henan 471934, P.R. China
}

Table 1: Data collection and handling.

\begin{tabular}{ll}
\hline Crystal: & Block, clear light colourless \\
Size: & $0.21 \times 0.18 \times 0.18 \mathrm{~mm}$ \\
Wavelength: & Mo $K \alpha$ radiation $(0.71073 \AA)$ \\
$\mu:$ & $0.54 \mathrm{~mm}^{-1}$ \\
Diffractometer, scan mode: & Bruker SMART, $\varphi$ and $\omega$-scans \\
$\theta_{\max }$, completeness: & $26^{\circ},>99 \%$ \\
$N(h k l)_{\text {measured }}, N(h k l)_{\text {unique }}, R_{\text {int }}:$ & $14833,5266,0.052$ \\
Criterion for $I_{\text {obs }}, N(h k l)_{\mathrm{gt}}:$ & $I_{\text {obs }}>2 \sigma\left(I_{\text {obs }}\right), 3374$ \\
$N(\text { param })_{\text {refined }}:$ & 385 \\
Programs: & Bruker [1], SHELX [2], OLEX2 [3]
\end{tabular}

steel vessel and heated at $140{ }^{\circ} \mathrm{C}$ for 4 days, and then cooled to room temperature over $60 \mathrm{~h}$. Colorless block crystals of 1 were obtained. Anal. (\%) Calcd. for $\mathrm{C}_{32} \mathrm{H}_{28} \mathrm{MnN}_{4} \mathrm{O}_{5}$ : C, 63.69, H 4.68, N 9.28. Found: C 63.74, H 4.62, N 9.31. IR $\left(\mathrm{cm}^{-1}\right)$ : 3127 m, $2958 \mathrm{~m}, 1601 \mathrm{~m}, 1565 \mathrm{~m}, 1531 \mathrm{~s}, 1459 \mathrm{~m}, 1342 \mathrm{~s}, 1092 \mathrm{~s}, 885 \mathrm{~s}$, $736 \mathrm{~s}$.

\section{Experimental details}

Hydrogen atoms were placed in their geometrically idealized positions and constrained to ride on their parent atoms.

\section{Discussion}

In recent years, a great deal of attention has focused on the self-assembly by metal-ligand interaction owing to luminescence, heterogeneous catalysis, gas storage/separations, and magnetic and electronic applications [4-7]. Although a lot of coordination polymers have been synthesized and characterized, precision synthesis of targeted structures has still been difficult $[8,9]$. Among the plenty of ligands employed in the fabrication of coordination polymers, the multidentate $\mathrm{N}$ - and/or $\mathrm{O}$-donor building blocks have received extensive attention [10]. For example, isophthalate and its derivatives with specific conformations and versatile coordination fashions can bind metal centers of specific coordination geometry to construct coordination polymers [11]. It is well known that the N-heterocyclic pyridine based organic molecules are also excellent ligands [12]. As an important polypyridyl ligand, 4'-(4-pyridyl)-2,2':6 $6^{\prime}, 2^{\prime \prime}$-terpyridine (PYTPY) has been widely used to build supramolecular architectures because of its excellent coordinating ability and large conjugated system that may provide potential supramolecular recognition sites. 
Table 2: Fractional atomic coordinates and isotropic or equivalent isotropic displacement parameters $\left(\AA^{2}\right)$.

\begin{tabular}{|c|c|c|c|c|}
\hline Atom & $x$ & $y$ & $z$ & $U_{\text {iso }} * / U_{\text {eq }}$ \\
\hline Mn1 & $0.32956(4)$ & $0.34435(2)$ & $0.25870(4)$ & $0.02996(14)$ \\
\hline 01 & $0.3152(3)$ & $0.38997(6)$ & $0.40596(19)$ & $0.0527(6)$ \\
\hline 02 & $0.2879(3)$ & $0.32899(6)$ & $0.50268(19)$ & $0.0580(6)$ \\
\hline 03 & $0.3746(3)$ & $0.32855(6)$ & $1.0055(2)$ & $0.0645(7)$ \\
\hline 04 & $0.3422(2)$ & $0.38833(6)$ & $1.10730(18)$ & $0.0457(5)$ \\
\hline N1 & $0.0772(2)$ & $0.32335(6)$ & $0.1787(2)$ & $0.0317(5)$ \\
\hline $\mathrm{N} 2$ & $0.3260(2)$ & $0.27326(6)$ & $0.25449(19)$ & $0.0277(5)$ \\
\hline N3 & $0.5802(2)$ & $0.32102(6)$ & $0.3354(2)$ & $0.0327(5)$ \\
\hline N4 & $0.3043(3)$ & $0.04529(7)$ & $0.2535(3)$ & $0.0548(7)$ \\
\hline C1 & $-0.0435(3)$ & $0.35075(8)$ & $0.1414(3)$ & $0.0373(7)$ \\
\hline $\mathrm{H} 1$ & -0.0233 & 0.3802 & 0.1520 & $0.045^{*}$ \\
\hline $\mathrm{C} 2$ & $-0.1962(3)$ & $0.33741(9)$ & $0.0882(3)$ & $0.0419(7)$ \\
\hline $\mathrm{H} 2$ & -0.2777 & 0.3575 & 0.0634 & 0.050 * \\
\hline C3 & $-0.2271(3)$ & $0.29419(9)$ & $0.0720(3)$ & $0.0395(7)$ \\
\hline H3 & -0.3299 & 0.2844 & 0.0357 & $0.047^{*}$ \\
\hline $\mathrm{C} 4$ & $-0.1034(3)$ & $0.26518(8)$ & $0.1104(3)$ & $0.0377(7)$ \\
\hline $\mathrm{H} 4$ & -0.1216 & 0.2356 & 0.0997 & $0.045^{*}$ \\
\hline $\mathrm{C} 5$ & $0.0471(3)$ & $0.28071(8)$ & $0.1648(2)$ & $0.0295(6)$ \\
\hline $\mathrm{C} 6$ & $0.1886(3)$ & $0.25213(8)$ & $0.2100(2)$ & $0.0295(6)$ \\
\hline $\mathrm{C} 7$ & $0.1818(3)$ & $0.20785(8)$ & $0.2095(2)$ & $0.0333(7)$ \\
\hline $\mathrm{H} 7$ & 0.0848 & 0.1939 & 0.1805 & 0.040 * \\
\hline $\mathrm{C} 8$ & $0.3196(3)$ & $0.18380(8)$ & $0.2520(3)$ & $0.0321(6)$ \\
\hline $\mathrm{C} 9$ & $0.4619(3)$ & $0.20636(8)$ & $0.2954(3)$ & $0.0341(7)$ \\
\hline H9 & 0.5572 & 0.1915 & 0.3230 & 0.041 * \\
\hline C10 & $0.4599(3)$ & $0.25078(8)$ & $0.2969(2)$ & $0.0284(6)$ \\
\hline C11 & $0.6053(3)$ & $0.27809(8)$ & $0.3439(2)$ & $0.0284(6)$ \\
\hline C12 & $0.7541(3)$ & $0.26100(8)$ & $0.3957(3)$ & $0.0381(7)$ \\
\hline $\mathrm{H} 12$ & 0.7690 & 0.2313 & 0.4022 & $0.046^{*}$ \\
\hline C13 & $0.8806(3)$ & $0.28876(9)$ & $0.4380(3)$ & $0.0416(7)$ \\
\hline H13 & 0.9818 & 0.2778 & 0.4739 & $0.050^{*}$ \\
\hline C14 & $0.8565(3)$ & $0.33236(9)$ & $0.4269(3)$ & $0.0425(7)$ \\
\hline H14 & 0.9405 & 0.3516 & 0.4536 & $0.051^{*}$ \\
\hline C15 & $0.7050(3)$ & $0.34694(8)$ & $0.3754(3)$ & $0.0378(7)$ \\
\hline H15 & 0.6883 & 0.3766 & 0.3679 & $0.045^{*}$ \\
\hline C16 & $0.3164(3)$ & $0.13605(8)$ & $0.2522(3)$ & $0.0333(6)$ \\
\hline C17 & $0.4337(3)$ & $0.11136(9)$ & $0.3376(3)$ & $0.0432(8)$ \\
\hline $\mathrm{H} 17$ & 0.5202 & 0.1247 & 0.3964 & $0.052^{*}$ \\
\hline C18 & $0.4209(4)$ & $0.06732(9)$ & $0.3346(3)$ & $0.0549(9)$ \\
\hline $\mathrm{H} 18$ & 0.5002 & 0.0517 & 0.3942 & $0.066^{*}$ \\
\hline C19 & $0.1942(4)$ & $0.06897(9)$ & $0.1717(3)$ & $0.0535(9)$ \\
\hline H19 & 0.1106 & 0.0546 & 0.1127 & $0.064^{*}$ \\
\hline $\mathrm{C} 20$ & $0.1943(3)$ & $0.11315(8)$ & $0.1674(3)$ & $0.0420(7)$ \\
\hline $\mathrm{H} 20$ & 0.1122 & 0.1277 & 0.1075 & $0.050^{*}$ \\
\hline C21 & $0.2988(3)$ & $0.36866(9)$ & $0.5055(3)$ & $0.0354(7)$ \\
\hline $\mathrm{C} 22$ & $0.2978(3)$ & $0.39241(8)$ & $0.6334(3)$ & $0.0311(6)$ \\
\hline $\mathrm{C} 23$ & $0.3207(3)$ & $0.37020(8)$ & $0.7539(2)$ & $0.0315(6)$ \\
\hline $\mathrm{H} 23$ & 0.3281 & 0.3402 & 0.7545 & $0.038^{*}$ \\
\hline C24 & $0.3328(3)$ & $0.39225(8)$ & $0.8740(2)$ & $0.0301(6)$ \\
\hline $\mathrm{C} 25$ & $0.3202(3)$ & $0.43703(7)$ & $0.8709(3)$ & $0.0316(6)$ \\
\hline $\mathrm{H} 25$ & 0.3270 & 0.4518 & 0.9514 & $0.038^{*}$ \\
\hline C26 & $0.2978(3)$ & $0.46025(8)$ & $0.7518(2)$ & $0.0303(6)$ \\
\hline $\mathrm{C} 27$ & $0.2867(3)$ & $0.43691(8)$ & $0.6334(3)$ & $0.0346(7)$ \\
\hline $\mathrm{H} 27$ & 0.2713 & 0.4517 & 0.5518 & $0.042^{*}$ \\
\hline $\mathrm{C} 28$ & $0.3519(3)$ & $0.36764(8)$ & $1.0037(3)$ & $0.0326(6)$ \\
\hline C29 & $0.2939(3)$ & $0.50960(8)$ & $0.7483(3)$ & $0.0375(7)$ \\
\hline
\end{tabular}

Table 2 (continued)

\begin{tabular}{lrrrr}
\hline Atom & $\boldsymbol{x}$ & $\boldsymbol{y}$ & $\boldsymbol{z}$ & $\boldsymbol{U}_{\text {iso }}{ }^{*} \boldsymbol{U}_{\text {eq }}$ \\
\hline C30 & $0.1494(4)$ & $0.52558(9)$ & $0.6386(3)$ & $0.0545(9)$ \\
H30A & 0.1574 & 0.5163 & 0.5511 & $0.082^{*}$ \\
H30B & 0.1454 & 0.5565 & 0.6408 & $0.082^{*}$ \\
H30C & 0.0550 & 0.5139 & 0.6553 & $0.082^{*}$ \\
C31 & $0.4458(3)$ & $0.52508(9)$ & $0.7163(3)$ & $0.0546(9)$ \\
H31A & 0.5356 & 0.5129 & 0.7808 & $0.082^{*}$ \\
H31B & 0.4511 & 0.5560 & 0.7215 & $0.082^{*}$ \\
H31C & 0.4458 & 0.5160 & 0.6266 & $0.082^{*}$ \\
C32 & $0.2854(4)$ & $0.52938(8)$ & $0.8830(3)$ & $0.0512(9)$ \\
H32A & 0.1914 & 0.5196 & 0.9048 & $0.077^{*}$ \\
H32B & 0.2832 & 0.5603 & 0.8756 & $0.077^{*}$ \\
H32C & 0.3764 & 0.5207 & 0.9533 & $0.077^{*}$ \\
O5 & $0.1275(3)$ & $0.45613(9)$ & $1.1520(3)$ & $0.0874(9)$ \\
H5A & 0.2072 & 0.4409 & 1.1494 & $0.131^{*}$ \\
H5B & 0.1490 & 0.4656 & 1.2327 & $0.131^{*}$ \\
\hline \multicolumn{5}{c}{}
\end{tabular}
new molecular selfassembly architectures.

The X-ray single-crystal structural analysis reveals that the complex is a one-dimensional chain coordination polymer. The asymmetric unit of the title structure contains one $\mathrm{Mn}$ (II) ion, one tbip $^{2-}$ anion, one PYTPY ligand and one lattice water molecule. The $\mathrm{Mn}(\mathrm{II})$ center is fivecoordinated via two oxygen atoms $(\mathrm{Mn1}-01=2.093(2) \AA$ and Mn1-04\#1 = 2.0876(19) $\AA$, symmetry code: \#1 $x, y, z-1$ ) derived from two different tbip ${ }^{2-}$ ligands and three nitrogen atoms $(\mathrm{Mn} 1-\mathrm{N} 1=2.244(2) \AA, \mathrm{Mn} 1-\mathrm{N} 2=2.202(2) \AA$, and $\mathrm{Mn} 1-\mathrm{N} 3=2.249(2) \AA$ ) from one PYTPY ligand in a distorted tetragonal pyramidal coordination geometry, which are normal bond distances found in other Mn complexes [13, 14]. The completely deprotonated tbip ${ }^{2-}$ ligand shows a monodentate coordination mode. Adjacent Mn(II) atoms are bridged by tbip $^{2-}$ ligands to form a single-chain structure, with the intrachain $\mathrm{Mn}-\mathrm{Mn}$ distance in the one-dimensional chain to be 10.229 A. The coordinated PYTPY ligands are attached on one side of this chain. The neighboring one-dimensional chains are connected into two-dimensional structure via hydrogen bonding interactions among the uncoordinated carboxyl groups, lattice water molecules and uncoordinated pyridine nitrogen atoms.

Acknowledgements: This work was supported by the National Natural Science Foundation of China (No. 21671114).

\section{References}

1. Bruker. APEX2, SAINT and SADABS. Bruker AXS Inc., Madison, WI, USA (2012). 
2. Sheldrick, G. M.: SHELXT-integrated space-group and crystal-structure determination. Acta Crystallogr. C71 (2015) 3-8.

3. Dolomanov, O. V.; Bourhis, L. J.; Gildea, R. J.; Howard, J. A. K.; Puschmann, H.: OLEX2: a complete structure solution, refinement and analysis program. J. Appl. Crystallogr. 42 (2009) 339-341.

4. Cui, Y. J.; Yue, Y. F.; Qian, G. D.; Chen, B. L.: Luminescent functional metal-organic frameworks. Chem. Rev. 112 (2012) 1126-1162.

5. Kitagawa, S.; Kitaura, R.; Noro, S. I.: Functional porous coordination polymers. Angew. Chem. Int. Ed. 43 (2004) 2334-2375.

6. Wu, C. D.; Hu, A. G.; Zhang, L.; Lin, W. B.: A homochiral porous metal-organic framework for highly enantioselective heterogeneous asymmetric catalysis. J. Am. Chem. Soc. 127 (2005) 8940-8941.

7. Gong, L. L.; Yao, W. T.; Liu, Z. Q.; Zheng, A. M.; Li, J. Q.; Feng, X. F.; Ma, L. F.; Yan, C. S.; Luo, M. B.; Luo, F.: Photoswitching storage of guest molecules in metal-organic framework for photoswitchable catalysis: exceptional product, ultrahigh photocontrol, and photomodulated size selectivity. J. Mater. Chem. A. 5 (2017) 7961-7967.

8. Li, C. P.; Zhao, X. H.; Chen, X. D.; Yu, Q.; Du, M.: Metal-involved solvothermal interconversions of pyrazinyl substituted azole derivatives: controllability and mechanism. Cryst. Growth Des. 10 (2010) 5034-5042.
9. Wang, H. H.; Yang, H. Y.; Shu, C. H.; Chen, Z. Y.; Wang, Y. Y.: Five new $\mathrm{Cd}$ (II) complexes induced by reaction conditions and coordination modes of 5-(1H-tetrazol-5-yl)isophthalic acid ligand: structures and luminescence. Cryst. Growth Des. 16 (2016) 5394-5402.

10. Guan, G. X.; Liu, X.; Yue, Q.; Gao, E. Q.: Homochiral metalorganic frameworks embedding helicity based on a semirigid alanine derivative. Cryst. Growth Des. 18 (2018) 364-372.

11. Jennifer, S. J.; Jana, A. K.: Influence of pyrazine/piperazine based guest molecules in the crystal structures of uranyl thiophene dicarboxylate coordination polymers: structural diversities and photocatalytic activities for the degradation of organic dye. Cryst. Growth Des. 17 (2017) 5318-5329.

12. Beves, J. E.; Constable, E. C.; Decurtins, S.; Dunphy, E. L.; Housecroft, C. E.; Keene, T. D.; Neuburger, M.; Schaffner, S.; Zampese, J. A.: Structural diversity in the reactions of $4^{\prime}$-(pyridyl)-2, $2^{\prime}: 6^{\prime}, 2^{\prime \prime}$-terpyridine ligands and bis $\left\{4^{\prime}\right.$-(4pyridyl)-2, 2':6', $2^{\prime \prime}$-terpyridine\}iron(II) with copper(II) salts. CrystEngComm 11 (2009) 2406-2416.

13. Merrill, C. A.; Cheetham, A. K.: Inorganic-organic framework structures; $\mathrm{M}(\mathrm{II})$ ethylenediphosphonates $(\mathrm{M}=\mathrm{Co}, \mathrm{Ni}, \mathrm{Mn})$ and a $\mathrm{Mn}$ (II) ethylenediphosphonato-phenanthroline. Inorg. Chem. 46 (2007) 278-284.

14. Ma, L. F.; Han, M. L.; Qin, J. H.; Wang, L. Y.; Du, M.: Mn" coordination polymers based on bi-, tri-, and tetranuclear and polymeric chain building units: crystal structures and magnetic properties. Inorg. Chem. 51 (2012) 9431-9442. 